\title{
Shoaling preference and social partner selection - behavioral, metabolic and psychiatric relevance in a zebrafish (Danio rerio) study
}

\author{
Christian Lenzi, Alin Ciobîcă, Mircea Nicoară, Gabriel Ionuț Plăvan, \\ Alexandrina-Ştefania Curpan, Daniel Timofte, Ştefan-Adrian Strungaru
}

Christian Lenzi - Associazione Eticoscienza, Turin, Italy

Alin Ciobîcă - "Alexandru Ioan Cuza" University of Iasi, Department of Research, Faculty of Biology, Iasi, România, Academy of Romanian Scientists, Bucuresti, România, Center of Biomedical Research, Romanian Academy, Iaşi, România

Mircea Nicoară - "Alexandru Ioan Cuza" University of Iasi, Department of Biology, Faculty of Biology, Iaşi, Romania, Doctoral School of Geosciences, Faculty of Geography-Geology, Iaşi, Romania

Gabriel Ionuţ Plăvan - “Alexandru Ioan Cuza” University of Iasi, Department of Biology, Faculty of Biology, Iasi, Romania,

Alexandrina-Ştefania Curpan - “Alexandru Ioan Cuza” University of Iasi, Department of Biology, Faculty of Biology, Iaşi, Romania,

Ştefan-Adrian Strungaru - "Alexandru Ioan Cuza" University of Iasi, Department of Research, Faculty of Biology, Iaşi, România

Daniel Timofte - "Grigore T. Popa" University of Medicine and Pharmacy, 16th Universitatii Street, 700115 Iaşi, Romania

\begin{abstract}
The zebrafish (Danio rerio) is considered an experimental model organism, with numerous applications. The mechanisms underlying shoaling preferences are complex, and some studies documented sex-related differences in social partner selection, little is known about the behavioral response to artificial visual stimuli representing conspecifics. The aim of this research was to evaluate the shoaling preference in zebrafish between two sex-shaped superstimuli, social non-moving 2D images were created using a graphics software. For this study, 20 sexually-mature individuals (males, $\mathrm{N}=10$, and females, $\mathrm{N}=10$ ) underwent 5 non-
\end{abstract}


r.\%\% invasive behavioral tests and, for each animal, ethological measurements were recorded using EthoVision XT video-tracking software. The findings showed statistically significant differences on zebrafish behavioral response. All individuals, without sex discrimination, tended to shoal more at the contact with the " 3 big females" stimulus and less with the " 1 big female", but only female zebrafish showed a preference for same social stimulus and also, spent more time in the proximity of the "big male" image rather than the "small male". We concluded that conspecifics represented by 2D artificial stimuli are efficient in inducing a behavioral response in this species. Moreover, we validate the existence of sex-dependent discrepancies regarding the shoaling preferences, as well as discussion how the aforementioned data has a possible psychiatric and metabolic relevance in this context.

\section{KEYWORDS}

Zebrafish, shoaling preference, social behavior, EthoVision XT, artificial stimuli.

\section{INTRODUCTION}

The zebrafish, Danio rerio, is a freshwater fish belonging to the Cyprinidae family (1). It is a valuable animal model for the study of several human diseases due to its various advantages (such as sequenced genome, ease of management, high reproduction rate). The importance of this species in scientific research is in a continuous increase $(2,3,4)$.

In social and sexual interactions involving this species, chemical communication seems to play an important role. In fact, it has been previously shown that females produce pheromones with an attractive effect on males (5). Pheromones are also important for ovulation, due to the male gonadal production (6) an exposure of only seven hours to a male it is enough for the female to lay eggs the following day (7). If females are kept in isolation for too long or they are old age a pathological state called "egg-bound" (i.e. accumulation of eggs in necrosis) can be encountered, which in some cases can be lethal. In order to prevent this state, females should be regularly exposed to males with accompanying reproductive events (8).

Furthermore, chemical communication alongside together with visual cues, comes into play in inter-individual recognition (9), for example for kin recognition (10).

However, amongst all sensory systems, the one best studied in zebrafish (for example for the shoaling preference) is the visual system (11). The recognition of visual cues and certain physical characteristics may be very important for this species, especially when it comes to shoaling preference (12). Moreover, some studies have documented sex-related differences in social partner's selection (13, 14).

In recent years, more and more studies have started to focus on the behavioral responses of different animal species, using various types of social stimuli, from videos with animated images to three-dimensional models $(15,16)$. As for zebrafish, an interesting study was carried out by Saverino and Gerlai in 2008. In this research, the authors investigated zebrafish's response to conspecifics that differ in coloration and / or shoaling tendencies, using computer-animated 2D stimuli. The results revealed, for example, a preference for the yellow phenotype and an avoidance of elongated zebrafish images (17).

In the recent years the behavioral response to social 2D non-moving images representing female "superstimuli" has become a topic of 
s

interest and was examined. A computergenerated visual stimulus representing a super female (with an evident abdomen region) were tested and significant sex-related differences in zebrafish responses were observed. In particular, females showed a statistically significant preference for 2D images of phenotypically less similar individuals (18).

In the present work, we wanted to investigate the shoaling preference to, graphically elaborated 2D fixed images, representing both female and male "superstimuli". Specifically, we wanted to test if a "super female" with an enlarged abdomen (comparable to the eggbound condition) could trigger a shoaling attraction within male or female zebrafish. Likewise, we wanted to study the behavioral response to a "super male", with a stretched caudal fin. The hypothesis was that males could be attracted to females with an evident abdominal region, which is a potential index of fertility. Similarly, even if it was never documented in zebrafish before, the elongated caudal fin could be an attractive trait for zebrafish females in sexual selection, as observed in other fish species (19).

In addition, the goal was also to expand the shoaling preference knowledge in order to develop new methodologies that could allow the use of virtual stimuli, minimizing the use of animals in experimental studies.

\section{MATERIALS AND METHODS}

\section{Ethics statement}

For this research all the animals were maintained and treated according to EU Commission Recommendation of 18 June 2007 on guidelines for the accommodation and care of animals used for experimental purposes, and of the European Parliament and Council from 22 September 2010 on the protection of animals used for scientific purposes $(20,21)$.

\section{Animals and housing}

The experiments were carried out at the "Ecotoxicology and Animal Behavior Laboratory", Faculty of Biology of the "Alexandru Ioan Cuza University" (Iasi, Romania).

A total of 20 sexually-mature GloFish ${ }^{\circledR}$ zebrafish (males, $\mathrm{N}=10$, and females, $\mathrm{N}=10$ ) were randomly selected from a captive group of $120 \mathrm{kept}$ in the same lab. All the animals were previously brought from different breeding facilities in order to maintain, as much as possible, a high genetic variability of the samples.

The animals were divided into two groups, one for males and one for females. The sex identification was performed through the recognition, for female individuals, of a more rounded and pronounced abdominal region, so no identifying labels were used. Each group was kept isolated for 48 hours (pre-test period) in a glass aquarium of $19.5 \times 17 \times 30$ cm (width $\mathrm{x}$ depth $\mathrm{x}$ height) with 5.00 liters of water, and an aerator. The water variables (temperature, $\mathrm{pH}$, and salinity) were monitored and keep constant, both in the pretest and experimental period. The animals were fed twice daily using commercial tropical fish food (Amtra Prima Flake" of Croci S.p.A). A 12:12 hours light / dark cycle was maintained with lights on at 7 am and off at $7 \mathrm{pm}$.

After the pre-test period, each animal underwent several behavioral tests using different artificial stimuli.

Experimental setting

The experimental environment was a multipurpose cross-maze adapted to a T-maze by 


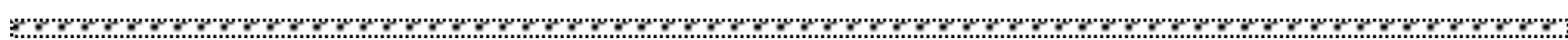

placing a dark panel that prevents the access to the top area. In this region, an adequate aquarium aerator was placed in order to maintain oxygen concentration constant throughout the observations (Supplementary Fig. S1).

A digital camera was placed above the center of the T-maze in order to record all the movements and behavioral variables of each animal. The camera was directly connected to a video-tracking software, EthoVision XT 11.5 (Noldus Information Technology), in the computer.

The artificial stimuli used during the experiments were graphically manipulated 2D images, using Adobe Photoshop CC 2017 (Supplementary Fig. S2). Starting from two pictures, one of male and one of female zebrafish, we created social "superstimuli". In particular, we built a "super female", with an evident abdominal region comparable to the egg-bound condition, and a "super male", with a stretched caudal fin (similar to the one of other fish species). Only the selected parts were modified: the body color and general morphometrically dimensions were maintained. For each 2D stimulus we built two versions: one standard (4 $\mathrm{cm}$ width) and one smaller ( $2.5 \mathrm{~cm}$ width). We already testes the same female stimulus in a previous study (18).

The artificial social stimuli were non-moving and fixed on the Plexiglas panels of the arms inside the T-maze.

\section{Behavioral tests}

In each behavioral test two different stimuli were used, one for each arm. During the trails the observed individual was carefully placed at the start-point and, after $1 \mathrm{~min}$ of habituation, the Plexiglas panel was removed. From that point onward, the video-tracking software recorded all movements and behavioral variables for 4 minutes for each test.

During the experiments, 5 non-invasive behavioral tests were carried out. Each fish underwent all different tests. To avoid habituation to the experimental setting that could affect the results, for each animal we change the order of the test and the position of the stimuli in the arms. For all behavioral tests two recorded trials were conducted.

First behavioral test: "big female" vs. "3 big females". In this behavioral we aimed to evaluate the shoaling preference of each zebrafish between one or three "super female" stimuli of standard dimension.

Second behavioral test: "big female" vs. "small female". We wanted to test the possible preference for a "super female" of standard dimensions or the smaller one. The two 2D stimuli differed only in width, not in shape.

Third behavioral test: "big female" vs. "small male". In the present behavioral test, we wanted to study the shoaling response towards two stimuli, the "super female" of standard dimensions and small "super male". So, the stimuli differed both in width and sex.

Fourth behavioral test: "big female" vs. "big male". In this behavioral test, we investigated the zebrafish social response to a "super female" and a "super male" stimuli. The 2D images represented conspecifics of standard dimensions but different sex.

Fifth behavioral test: "big male" vs. "small male". Lastly, we wanted to examine the shoaling preference between two "super male" stimuli: one of standard dimensions and one smaller. Both 2D images represented male zebrafish with stretched caudal fin. 


\section{Statistical analysis}

All statistical behavioral data obtained from the experimental tests were analyzed using the OriginPro 2016 statistical software. The variables recorded for each group are: cumulative duration and mean duration. Cumulative duration is the total time spent in one choice area and it is express in percentage (\%). The mean duration is the average of time spent in one choice area and it is express in seconds (s).

For all behavioral tests, the normal data distribution was evaluated by KolmogorovSmirnov test and for normal distributed samples the Student's t-test was used, while for samples not normally distributed, we used Mann-Whitney U test and Wilcoxon rankings test. Moreover, homoscedasticity was analyzed (Levene test), and to compare the means post hoc analyzes were performed (Bonferroni test and Tukey test).

In the first, "big female vs. 3 big females", and in the second behavioral test, "big female vs. small female", Student's t-test was used for both the variables.

In the third behavioral test, "big female vs. small male", Student's t-test was used for the cumulative duration (within the female sample and in the comparison for the "big female" area) and for the mean duration (within the male sample and in the comparison for the "big female" zone). The Mann-Whitney $U$ test was used in the comparison for the "small male" area cumulative and mean duration. Lastly, the Wilcoxon rankings test was used for the cumulative duration of the male sample and the mean duration within the female sample.

In the fourth behavioral test, "big female vs. big male", Student's t-test was used for the cumulative duration (within the sample and in the comparison between them for both stimuli zones) and mean duration (within female sample and in the comparison for the "big male" stimulus). The Wilcoxon rankings test was used for the mean duration within the male sample. Lastly, we used the MannWhitney $\mathrm{U}$ test to compare the two samples for the "big female" area.

In the fifth and last behavioral test, "big male vs. small male", Student's t-test was used for both the variables.

In order to avoid confusion, it has been reported "mean (SD, Standard Deviation)" and not "mean \pm SD" given the similar notation, which is often found in the literature, for the standard error (22).

\section{RESULTS}

In the first behavioral test, "big female vs. 3 big females", both males and females shoaled more with the " 3 big females" stimulus, in terms of cumulative duration (males: Student's t-test, $\mathrm{t}=-2.696, \mathrm{~N} 1=10, \mathrm{~N} 2=10$, $\mathrm{p}=0.024$, females: Student's t-test, $\mathrm{t}=-2.466$, $\mathrm{N} 1=10, \mathrm{~N} 2=10, \mathrm{p}=0.036)$. No statistically significant differences were found in the comparison between male and female samples both for mean duration in the "big female" area (Student's t-test, $\mathrm{t}=1.204, \mathrm{~N} 1=10, \mathrm{~N} 2=$ $10, p=0.239$ ) and in the " 3 big females" area (Student's t-test, $\mathrm{t}=-2.135, \mathrm{~N} 1=10, \mathrm{~N} 2=10$, $\mathrm{p}=0.381)($ Fig. 1A).

In the second behavioral test, "big female vs. small female", there were no statistically significant differences between males and females, in terms of cumulative duration, for the time spent near the two stimulus zones, "big female" (Student's t-test, $\mathrm{t}=0.169, \mathrm{~N} 1=$ $10, \mathrm{~N} 2=10, \mathrm{p}=0.686)$ and "small female" (Student's t-test, $\mathrm{t}=0.116, \mathrm{~N} 1=10, \mathrm{~N} 2=10$, $p=0.737)$. We found the same also for the mean duration in the two areas, "big female" 
(Mann-Whitney test $\mathrm{U}, \mathrm{U}=38, \mathrm{Z}=-0.869$,

$\mathrm{N} 1=10, \mathrm{~N} 2=10, \mathrm{p}=0.385)$ and "small

female" (Student's t-test, $\mathrm{t}=2.295, \mathrm{~N} 1=10$,

$\mathrm{N} 2=10, \mathrm{p}=0.103)($ Fig. 1B).

In the third behavioral test, "big female vs. small male", there were no statistically significant differences between males and females, in terms of cumulative duration
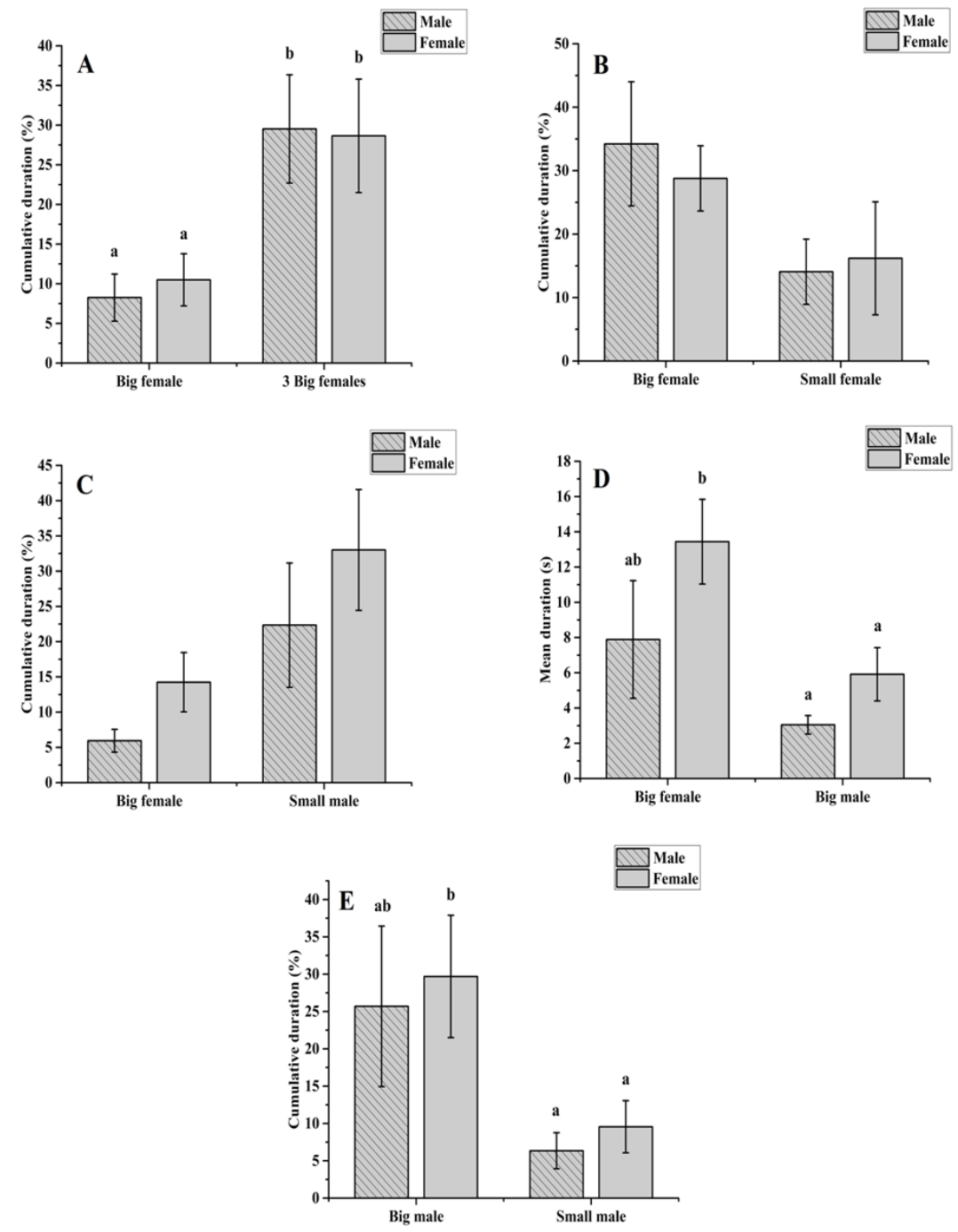

Figure. 1 Main results of the behavioral tests.

A. First behavioral: test (big female vs. 3 big females): cumulative duration of activity in the arm with stimuli (mean $\pm \mathrm{SD})$; same letter means "not statistically significant differences". $(* p<0.05)$. B. Second behavioral test (big female vs. 3 small females): cumulative duration of activity in the arm with stimuli (mean $\pm \mathrm{SD}),\left({ }^{*} \mathrm{p}<0.05\right)$. C. Third behavioral test (big female vs. small male): mean duration of activity in the arm with stimuli (mean $\pm \mathrm{SD})$; same letter means "not statistically significant differences". ( $\left.{ }^{*}<<0.05\right)$. D. Fourth behavioral test(big female vs. big male): mean duration in the arm with stimuli (mean $\pm \mathrm{SD}$ ); same letter means "not statistically significant differences" $\left({ }^{*} p<0.05\right)$. E. Fifth behavioral test (big male vs. small male): cumulative duration in the arm with stimuli (mean $\pm \mathrm{SD})$; same letter means "not statistically significant differences" $(* \mathrm{p}<0.05)$. 
In the fourth behavioral test, "big female vs. big male", females exhibited a preferrence for the "big female" rather than the "big male", in terms of mean duration (Student's t-test, $\mathrm{t}=$ 2.443, N1 = 10, N2 = 10, $\mathrm{p}=0.037)$. No statistically significant differences were found within the male sample, either in terms of cumulative duration (Student's t-test, $\mathrm{t}=$ 1.003, $\mathrm{N} 1=10, \mathrm{~N} 2=10, \mathrm{p}=0.342$ ) or the mean duration (test of signs by ranks of Wilcoxon, $\mathrm{W}=9, \mathrm{Z}=-1.504, \mathrm{~N} 1=10, \mathrm{~N} 1=$ 10, $\mathrm{p}=0.129)($ Fig. 1D).

In the fifth and last behavioral test, "big male vs. small male", females shoaled more in contact with the "big male" stimulus, in terms of cumulative duration (Student's t-test, $\mathrm{t}=$ 2.304, $\mathrm{N} 1=10, \mathrm{~N} 2=10, \mathrm{p}=0.047)$. No statistically significant difference was found within the male sample, neither for the cumulative duration (Student's $\mathrm{t}$-test, $\mathrm{t}=$ $1.698, \mathrm{~N} 1=10, \mathrm{~N} 2=10, \mathrm{p}=0.124$ ) nor for the mean (Student's t-test, $\mathrm{t}=1.315, \mathrm{~N} 1=10$, $\mathrm{N} 2=10, \mathrm{p}=0.266)($ Fig. 1E).

Additional, supplementary information about the experimental design and the obtained data can be found in Supplementary Figures 2 and 3:

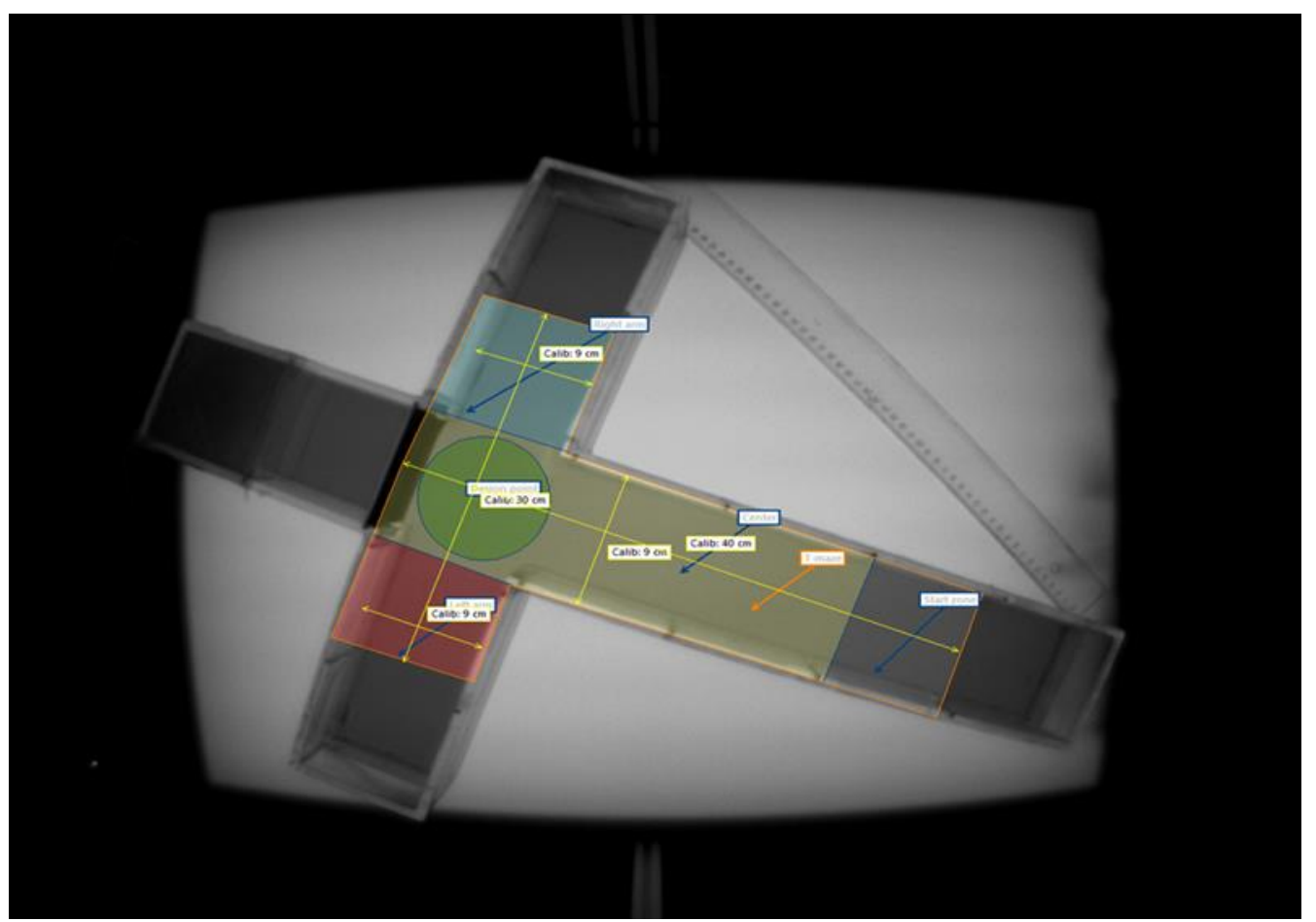

Figure 2 . Experimental setting. For the present study a multi-purpose cross-maze, adapted to a Tmaze, was used as experimental environment. The apparatus is a cross-shaped Plexiglas labyrinth, equipped with two arms, with a total volume of 5.00 liters. Here we present the "virtual maze" built and adapted to the real one. It is composed by a central corridor (in yellow) of $49 \times 9 \times 9 \mathrm{~cm}$ (width $\mathrm{x}$ depth $\mathrm{x}$ height), containing the start-point (transparent area with orange borders on the right of the image) and the decision-point (green circle between the two arms, on the left of the image). Transversely, there was another corridor of $49 \times 9 \times 9 \mathrm{~cm}$ (width $\times$ depth $\times$ height), representing the arms of choice (blue and red zones). Transparent separating panels were used to delimit the startpoint zone and the stimulus areas. 

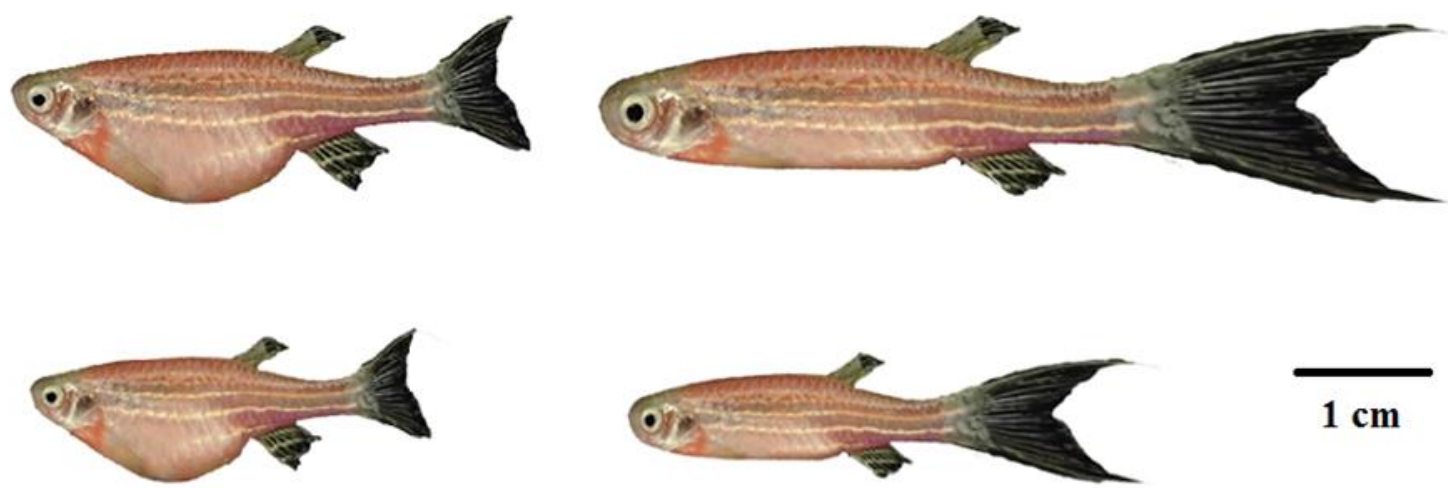

$1 \mathrm{~cm}$

Figure 3. The artificial stimuli used for the behavioral tests. Different 2D images were realized using photos of adult males and females that were manipulated by a graphics software. The nonmoving stimuli were placed in the choice areas of the experimental apparatus, fixed to a Plexiglas panel.

\section{DISCUSSION}

For the present study we wanted to test the zebrafish shoaling preference for social artificial stimuli. Starting from what was previously done by (17) we wanted to evaluate the behavioral response induced by "compressed" or "lengthened" individuals. In order to achieve this, we use two 2D graphically manipulated stimuli of different dimensions: one "super female" and one "super male). The first stimulus (with an exaggerated abdominal region) was already tested in our previous study (18), while here we used for the first time the "super male" image (with an elongated caudal fin). But unlike the previous study of (17), we maintained the color and all morphometric parameters; moreover, we opted for nonmoving stimuli.

In the first behavioral test, "big female vs. 3 big females", both males and females preferred to spend more time close to the " 3 big female" area. This result confirms what was previously observed in other studies, where generally zebrafish prefer to shoal with bigger rather than smaller groups $(8,23)$.
Both in the second, "big female vs. small female", and in the third behavioral test, "big female vs. small male", no statistically significant differences were found for shoaling preference (in terms of cumulative and mean duration) for both the male and female samples. These findings are in disagreement with what was claimed in one article by Bartolini and colleagues (24), where zebrafish seemed to prefer smaller artificial stimuli. But, the evidence emerged from the second behavioral test is in agreement with Pyron's previous observation regarding male sexual preferences based on body size (25).

In the fourth behavioral test, "big female vs. big male", the females showed a preference (in terms of mean duration) for the "big female" area. For males, no statistically significant difference was observed.

In the last behavioral test, "big male vs. small male", females showed a significant preference (in terms of cumulative duration) for the bigger male-shaped stimulus compared to the other one, while for males no choice prevailed on the other. Again, these results disagree with the preference for smaller artificial stimuli observed by Bartolini and colleagues (24). The preference for the "big 


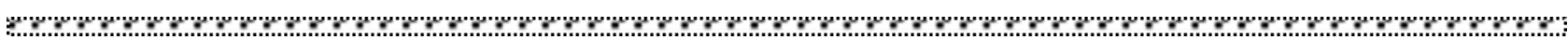

male" stimulus could be associated with a sexual attraction of the females towards a partner with greater body size. In any case, the choice towards a "big female" compared to a "big male" can be justified by the prevalence of the social stimulus of shoaling with the possibility of obtaining greater resources with respect to sexual and reproductive stimulation.

The lack of preference between a "big female" and a "small female" is an interesting result especially for the male sample in which it is clear that the factor of body size may not influence the choice of partner. However, it is difficult to interpret the absence of choice between the "big female" and the "small male" by the females.

Compared to the results of the fifth behavioral test, "big male vs. small male", these are in agreement with what was previously observed by Pyron, according to which females have a certain preference for males with greater body size (25). However, these same results are in disagreement with what emerges from the study of Spence and Smith (26) according to which the females would actively choose the partner without a preference determined by the body size or the rank of dominance.
Lastly, the results of the fifth test are in disagreement with what was observed in a previous study, where the zebrafish showed an avoidance regarding a manipulated artificial stimulus representing an elongated individual (17). Nonetheless, in that study, the general morphometric body dimensions were not maintained thus the results could not be really comparable.

Interestingly, in our study we showed that non-moving social stimuli can induce a shoaling attraction in zebrafish. The findings of this study confirm what was found in our recent study about "superstimuli" and zebrafish social response (18). Zebrafish show a statistically significant social preference for certain artificial stimuli, even if those are fixed. We assume the absence of any experimental effect affecting the results since: (a) each animal underwent all behavioral tests but in different order, and (b) the stimuli were located in different arms for each trial. It remains unclear whether the evident abdominal region or the elongated caudal fin could be determining factors for sexual selection in zebrafish. Further future research will be required to better understand this aspect.

\section{CONCLUSIONS}

In the present study, we concluded that our hypothesis was confirmed: 2D images representing social "superstimuli", even fixed and non-moving, can induce a behavioral response in terms of shoaling preference. The findings showed sex-related differences when it comes to social choices. In fact, even if both samples preferred the " 3 big females" rather than the " 1 big female", in general males were less selective for the shoaling preferences between different stimuli. Females, instead, showed a preference for the female social stimulus over the male-shaped one and spent more time close to the image of "super male" of standard dimensions rather than a smaller one. Regarding this last aspect, it has not been well clarified whether this choice should be associated with a reproductive preference or is purely a social choice (shoaling).

\section{ACKNOWLEDGEMENTS AND DISCLOSURES}

Funding: This work was supported by the Project Resources Pilot Center for cross-border preservation of the aquatic biodiversity of Prut River (MIS-ETC 1150 Romania-Ukraine-Republic 

of Moldova) and the author Alin Ciobică was receiving payment from UEFISCDI (PN-III-P1-1.1TE-2016-1210) during the time of the study.

All authors declare that there are no conflicts of interest for this study, except the grants described above.

\section{REFERENCES}

1. Hamilton F. An account of the fishes found in the river Ganges and its branches. In Edinburgh \& London: A. Constable and company; 1822. p. 1-405. Available from: http://www.biodiversitylibrary.org/bibliography/6897

2. Goldsmith P, Solari R. The role of zebrafish in drug discovery. Drug Discov world, 2003;Spring:74-8. Available from: https://www.ddw-online.com/drug-discovery/p148422-the-role-of-zebrafish-in-drug-discovery-spring-03.html

3. Robea M-A, Strungaru Ș-A, Lenzi C, Nicoară M, Ciobică A. The Importance of Rotenone in Generating Neurological and Psychiatric Features in Zebrafish-Relevance for a Parkinson's Disease Model. Acad Rom Sci - Ann Ser Biol Sci. 2018;7(1):55-67.

4. Robea M-A, Săvucă A, Strungaru Ș-A, Lenzi C, Grasso C, Plăvan G, et al. The general biological relevance of the oxidative stress status in replicating some neuropsychiatric and digestive - related manifestations in zebrafish. Rom Biotechnol Lett, 2019 Aug 20 (cited 2019 Nov 19);24(4):571-9. Available from: https://www.erepository.org/rbl/vol.24/iss.4/2.pdf

5. Bloom HD, Perlmutter A. A sexual aggregating pheromone system in the zebrafish,Brachydanio rerio (hamiltonbuchanan). J Exp Zool, 1977, 199(2):215-26. Available from: http://www.ncbi.nlm.nih.gov/pubmed/845579

6. Spence R, Smith C. Innate and Learned Colour Preference in the Zebrafish, Danio rerio. Ethology, 2008;114(6):582-8. Available from: http://doi.wiley.com/10.1111/j.1439-0310.2008.01515.x

7. Eaton RC, Farley RD. Spawning Cycle and Egg Production of Zebrafish, Brachydanio rerio, in the Laboratory. Copeia, 1974.28;1974(1):195. Available from: https://www.jstor.org/stable/1443023?origin=crossref

8. Spence R, Gerlach G, Lawrence C, Smith C. The behaviour and ecology of the zebrafish, Danio rerio. Biol Rev, 2008;83(1):13-34. Available from: http://www.ncbi.nlm.nih.gov/pubmed/18093234

9. Gerlach G, Lysiak N. Kin recognition and inbreeding avoidance in zebrafish, Danio rerio, is based on phenotype matching. Anim Behav, 2006; from: https://linkinghub.elsevier.com/retrieve/pii/S0003347206001096

10. Hinz C, Kobbenbring S, Kress S, Sigman L, Müller A, Gerlach G. Kin recognition in zebrafish, Danio rerio, is based on imprinting on olfactory and visual stimuli. Anim Behav. 2013;85(5):925-30. Available from: https://www.sciencedirect.com/science/article/pii/S0003347213000869

11. Guo S. Linking genes to brain, behavior and neurological diseases: what can we learn from zebrafish? Genes Brain Behav,2004;3(2):63-74. Available from: http://www.ncbi.nlm.nih.gov/pubmed/15005714

12. Spence R, Smith C. The Role of Early Learning in Determining Shoaling Preferences Based on Visual Cues in the Zebrafish, Danio rerio. Ethology. 2007;113(1):62-7. Available from: http://doi.wiley.com/10.1111/j.14390310.2006.01295.x

13. Snekser J, Ruhl N, Bauer K, Mcrobert S. The influence of sex and phenotype on shoaling decisions in zebrafish. Int J Comp Psychol. 2010. 1;23:70-81.

14. Lenzi C, Grasso C, Nicoara M, Savuca A, Ciobica A, Plavan G, et al. Influence of environmental colors and longterm sex isolation on zebrafish shoaling behavior. Arch Biol Sci, 2019;71(2):329-37. Available from: http://www.doiserbia.nb.rs/Article.aspx?ID=0354-46641900015L

15. Woo KL, Rieucau G. From dummies to animations: a review of computer-animated stimuli used in animal behavior studies. Behav Ecol Sociobiol, 2011;65(9):1671-85. Available from: http://link.springer.com/10.1007/s00265011-1226-y

16. Ruberto T, Mwaffo V, Singh S, Neri D, Porfiri M. Zebrafish response to a robotic replica in three dimensions. R Soc Open Sci, 2016;3(10):160505. Available from: http://www.ncbi.nlm.nih.gov/pubmed/27853566

17. Saverino C, Gerlai R. The social zebrafish: Behavioral responses to conspecific, heterospecific, and computer animated fish. Behav Brain Res,2008.5;191(1):77-87. Available from: http://www.ncbi.nlm.nih.gov/pubmed/18423643 18. Lenzi C, Grasso C, Nicoara M, Savuca A, Ciobica A, Plavan GI, et al. Behavioral responses of zebrafish (Danio rerio) to different 2D non-moving stimuli. Arch Biol Sci. 2020;72(1). 

19. Hernandez-Jimenez A, Rios-Cardenas O. Natural versus sexual selection: predation risk in relation to body size and sexual ornaments in the green swordtail. Anim Behav, 2012; 84(4):1051-9. Available from: https://linkinghub.elsevier.com/retrieve/pii/S0003347212003533

20. Russell WMS, Burch RL. The principles of humane experimental technique. Burch RL (Rex L, editor. London: Methuen; 1959.

21. Union E. Directive 2010/63/EU of the European Parliament and of the Council of 22 September 2010 on the protection of animals used for scientific purposes. Off J Eur Union. 2010;276:33-79.

22. Jaykaran. "Mean \pm SEM" or "Mean (SD)"? Indian J Pharmacol. 2010. 1;42:329.

23. Pritchard VL, Lawrence J, Butlin RK, Krause J. Shoal choice in zebrafish, Danio rerio: the influence of shoal size and activity. Anim Behav, 2001; 62(6):1085-8. Available from: https://www.sciencedirect.com/ science/article/pii/S0003347201918585

24. Bartolini T, Mwaffo V, Showler A, Macrì S, Butail S, Porfiri M. Zebrafish response to 3D printed shoals of conspecifics: the effect of body size. Bioinspir Biomim, 2016; 11(2):1-12. Available from: http://www.ncbi.nlm.nih.gov/pubmed/26891476

25. Pyron M. Female preferences and male--male interactions in zebrafish (Danio rerio). Can J Zool, 2003 Jan 1;81(1):122-5. Available from: https://doi.org/10.1139/z02-229

26. Spence R, Smith C. Mating preference of female zebrafish, Danio rerio, in relation to male dominance. Behav Ecol, 2006 Sep 1;17(5):779-83. Available from: http://academic.oup.com/beheco/article/17/5/779/207220/Matingpreference-of-female-zebrafish-Danio-rerio

\section{Correspondence:}

Daniel Timofte,

MD, PhD, Professor, "Grigore T. Popa" University of Medicine and Pharmacy, 16th Universitatii Street, 700115 Iasi, Romania, dantimofte@yahoo.com

Submission: 09 oct 2020

Acceptance: 02 dec 2020 